\title{
Two cases of Salmonella breast abscess in pregnancy: a diagnostic challenge
}

\author{
Aishwarya Parthasarathy*, Kundavi Shankar, Suresh Anand
}

\begin{abstract}
${ }^{1}$ FNB Reproductive Medicine Trainee, Institute of Reproductive Medicine, Madras Medical Mission, Chennai, Tamil Nadu, India

${ }^{2}$ Institute of Reproductive Medicine, Madras Medical Mission, Chennai, Tamil Nadu, India

${ }^{3}$ Breast and Oncoplastic Surgeon, Institute of Reproductive Medicine, Madras Medical Mission, Chennai, Tamil Nadu, India
\end{abstract}

Received: 14 April 2019

Revised: 30 May 2019

Accepted: 06 May 2019

\section{*Correspondence:}

Dr. Aishwarya Parthasarathy,

E-mail: seventhsensed@gmail.com

Copyright: (C) the author(s), publisher and licensee Medip Academy. This is an open-access article distributed under the terms of the Creative Commons Attribution Non-Commercial License, which permits unrestricted non-commercial use, distribution, and reproduction in any medium, provided the original work is properly cited.

\begin{abstract}
Breast abscess in pregnancy due to Salmonella has been very rarely reported in the literature and most of the cases till now are due to unknown risk factors or in otherwise immunocompromised adults. Localized disease can occur due to bacteremia followed by seeding of bacteria at distant sites. We report two cases of breast abscess in pregnancy cause by Salmonella typhi where drainage of abscess and appropriate antibiotics helped in complete resolution.
\end{abstract}

Keywords: Salmonella typhi, Breast abscess, Pregnancy

\section{INTRODUCTION}

Breast abscess usually occurs in the setting of lactation with Staphylococcus aureus being the most common cause. ${ }^{1}$ Salmonella is a Gram-negative bacteria which usually has intestinal preponderance. Extra intestinal salmenellosis occurs rarely in immunocompromised such as pregnancy; Human immunodeficiency virus; prolonged immunotherapy, advanced age and local infection. ${ }^{2,3}$ If the infection is untreated or resistant to treatment, circulating bacteria can settle in various organs and cause extraintestinal disease and many a times be misdiagnosed by lack of knowledge. ${ }^{4,5}$

\section{CASE REPORT}

\section{Case 1}

A 29 year old lady, G3P1L1A1, with previous cesarean section, came to us at $31+2$ weeks POG with high grade fever and pain in the left breast for 2 days preceded by several episodes of loose stools for 5 days. She was seen at the breast clinic and on examination a $4 \mathrm{~cm}$ lump was seen on the left breast, associated with warmth and tenderness. Further, ultrasound of the breast revealed diffuse tissue edema (U2) and no significant evidence of abscess. She was started on empiric antibiotics (Amoxicillin/clavulanic acid) and fever investigations were organized. As the symptoms of the patient increased in the next two days, she was admitted, and ultrasound of the breasts was repeated (Figure 1).

It revealed fluid collection within ducts suggestive of intraductal abscess, which was aspirated (Figure 2) and sent for culture and sensitivity testing.

Leucocyte count was normal with $79 \%$ polymorphs. In the microbiology lab, the pus sample was processed on Mac conkey Agar, Sheep blood agar and Thioglycollate broth. The blood and pus culture showed Salmonella typhi growth sensitive to Ampicillin, Azithromycin, Ceftrioxone, Chloramphenicol and Co-trimoxazole. Stool 
culture did not show growth of organisms. Subsequently, her 4 year old daughter also had fever was diagnosed with typhoid.

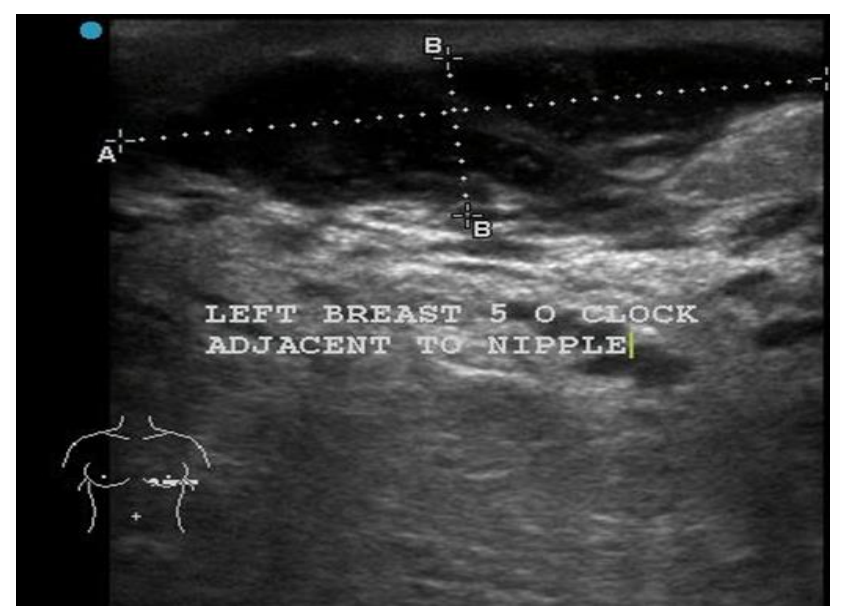

Figure 1: Left breast abscess ultrasound (USG) picture.

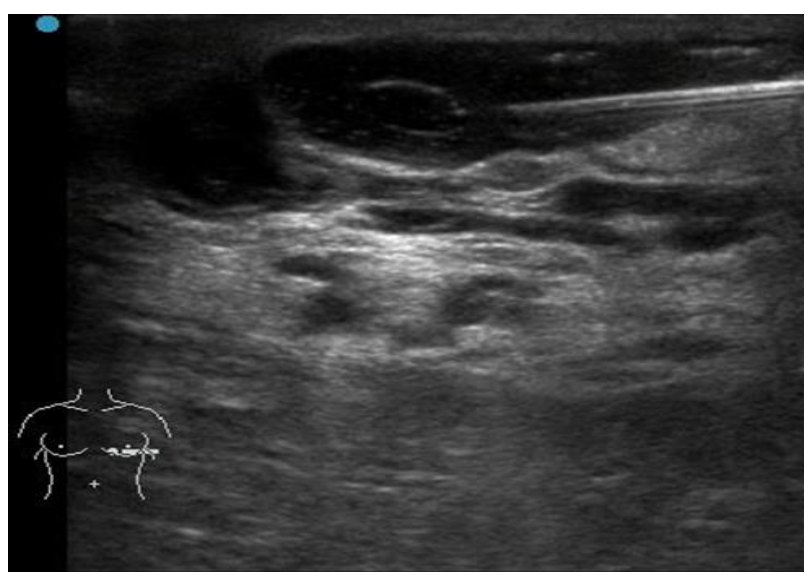

Figure 2: USG guided aspiration.

The patient was started on inj. Ceftrioxone 1 gram twice daily after the sensitivity testing After 48 hours of parenteral antibiotics, oral cefixime tablets were started and she became symptomatically better. The antibiotic course was continued for 21 days and symptoms subsided completely after a 12 days course of Cefixime. She delivered a healthy newborn at $40+1$ week by emergency LSCS and post partum period was uneventful with respect to lactation.

\section{Case 2}

A 26 year old, Primigravida at 35 weeks gestation was referred with a tender lump in the breast associated with fever for 5 days. She was treated with empirical intravenous antibiotics (Ceftrioxone, Metronidazole) with no relief of symptoms, elsewhere. Apart from gestational hypertension which was adequately controlled, she had no other issues in the pregnancy. Clinical examination revealed $6 \mathrm{~cm}$ of indurated lump with erythema, warmth and fluctuation in the right breast, suggestive of abscess. The contralateral breast was normal. Ultrasound of the right breast showed diffuse edema of subcutaneous tissues with marked duct ectasia and a $25.1^{*} 6.6 \mathrm{~mm}$ hypoechoic area within, suggestive of abscess (U2) (Figure 3) and $4 \mathrm{ml}$ of thick pus was drained was drained from the abscess (Figure 4) which showed growth of Salmonella typhi, sensitive to Ampicillin, Azithromycin, Ceftrioxone and Chloramphenicol. She was given oral Azithromycin for two weeks as she had an incomplete course of Ceftrioxone elsewhere. The patient became symptomatically better and the abscess completely resolved after two week.

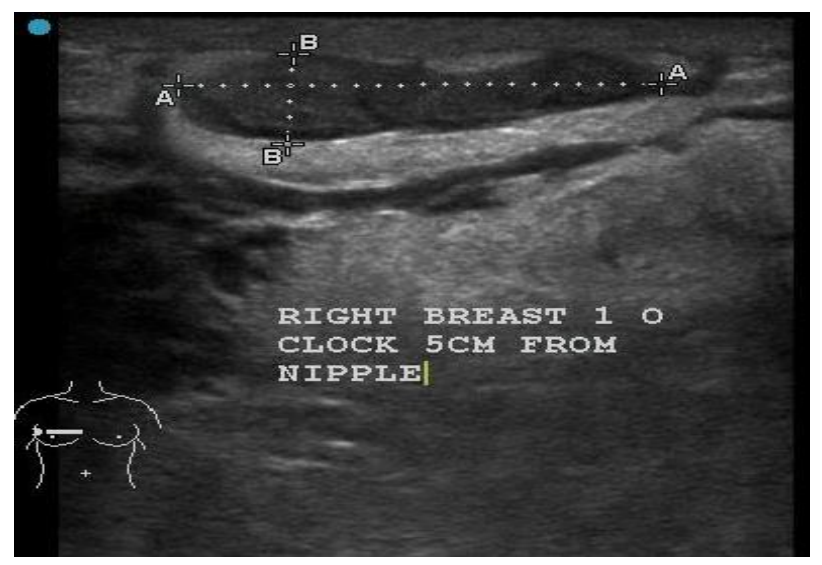

Figure 3: 25.1*6.6 mm hypoechoic area in right breast- abscess.

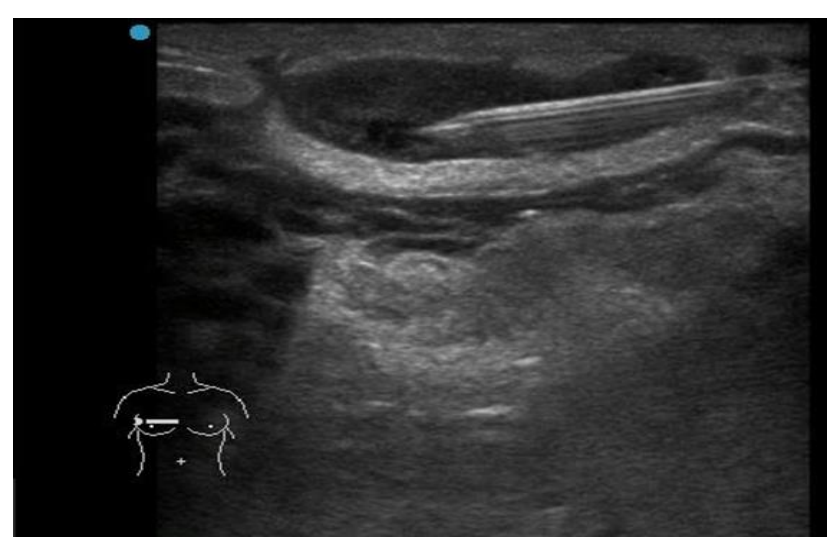

Figure 4: USG guided aspiration.

\section{DISCUSSION}

Salmonella breast abscess is a rare sequalae of enteric fever most commonly seen in the immunocompromised. ${ }^{6}$ Though said to be common in pregnancy, has not been reported widely. The literature search revealed that most such cases have been reported from India in non pregnant or lactating women. ${ }^{7-9}$ The organism can disseminate through hematogenous route and seed in the breast tissue. The usual presentation is febrile illness with loose stools. Both our patients have history of febrile illness but loose stools in only one case. Contact history should be clearly 
elicited as the daughter also subsequently developed the same infection, in the first case. Agarwal et al, have reported two cases where they had found evidence of loose stools on the retrospect. ${ }^{7}$

Delay in the diagnosis is inevitable as it's a rare diagnosis. However, prompt aspiration of the pus and adequate microbiological workup is the key to diagnosis. Recurrent, bilateral abscesses and antibiotic resistance seem to be a fairly common manifestation. ${ }^{6,10}$ This could point out towards the need for being selective about the antibiotic policy and avoid prolonged courses of empirical antibiotics.

Pregnancy is an immunocompromised state due to diminished cell mediated immunity and any infection must be treated vigorously. Initial empirical therapy and its failure bring to light the importance of microbiological culture of the local infection. As it poses a diagnostic dilemma, referral to an expert in the field, aspiration of pus and treatment with sensitive antibiotics will offer the best possible and prompt treatment of the condition. Follow up is essential as such patients have a possibility of recurrence.

\section{CONCLUSION}

A detailed history coupled with appropriate sampling, microbiological workup and treatment with sensitive antibiotics is the key to treatment as any infection in pregnancy could lead to serious feto-maternal compromise. One must always keep in mind the possibility of Salmonella breast abscess in a country like India.

Funding: No funding sources

Conflict of interest: None declared

Ethical approval: Not required

\section{REFERENCES}

1. Kataria K, Srivastava A, Dhar A. Management of lactational mastitis and breast abscesses: review of current knowledge and practice. Indian J Surg. 2013;75(6):430-5.

2. Ghadage DP, Wankhade AB, Mali RJ, Bhore A V. Recurrent breast abscess due to Salmonella paratyphi A: an unusual case. Int $J$ Res Med Sci. 2014;2(3):1236-8.

3. Sudhaharan S, Padmaja K, Solanki R, Lakshmi V, Umabala P, Aparna B. Extra-intestinal salmonellosis in a tertiary care center in South India. 2014;8(7):831-7.

4. Baran I, Aksu N, Aksoy A. Breast abscess due to Salmonella Typhimurium in a patient with rheumatoid arthritis: a case report. BMC Infect Dis. 2016;16(1):348.

5. Jayakumar K, Appalaraju B, Govindan VK. An atypical presentation of Salmonella typhi - a case report. Indian J Med Microbiol. 2003;21(3):211-2.

6. Singh G, Dasgupta M, Gautam V, Behera A, Ray P. Bilateral Breast Abscesses due to Salmonella Enterica Serotype Typhi. J Glob Infect Dis. 2011;3(4):402-4

7. Agrawal S, Yadav VS, Srivastava A, Kapil A, Dhawan B. Breast abscess due to Salmonella paratyphi A : Case reports with review of literature. Intractable rare Dis Res. 2018;7(2):130-3.

8. Sood S. Breast Abscess by Salmonella Paratyphi A: Case Report and Literature Review. J Clin Diagn Res. 2015;9(9):DD03-4.

9. Murugesan N, Samy RA, MSVAA. A RARE CASE OF BREAST ABSCESS DUE TO SALMONELLA TYPHI -. Int J Cur Res Rev. 2016;8(5):10-2.

10. Fernando S, Molland JG, Gottlieb T. Failure of oral antibiotic therapy, including azithromycin, in the treatment of a recurrent breast abscess caused by Salmonella enterica serotype Paratyphi A. Pathog Glob Health. 2012;106(6):366-9.

Cite this article as: Parthasarathy A, Shankar K, Anand S. Two cases of Salmonella breast abscess in pregnancy: a diagnostic challenge. Int J Reprod Contracept Obstet Gynecol 2019;8:2885-7. 